\title{
CONGESTION CONTROL BASED ON SLIDING MODE CONTROL AND SCHEDULING WITH PRIORITIZED QUEUING FOR WIRELESS NETWORKS
}

\author{
R.Keerthana ${ }^{1}$, S.Lavanya ${ }^{2}$ \\ ${ }^{I}$ PG Scholar, Dept. of CSE, Sona College of Technology, Salem-636005, TN, India \\ ${ }^{2}$ Asst. Professor, Dept. of CSE, Sona College of Technology, - 636005, TN, India
}

\begin{abstract}
The application of sliding mode control in wireless network is used for considering the joint congestion control and scheduling. Incorporating dual decomposition enables the joint congestion control and scheduling problem to be considered separately. The communication among these two sub problems is given by the lagrangian price value. Based on the utility optimization of network the congestion control performance for queues can be improved using sliding mode based congestion controller. Buffers on network devices are managed with various queuing techniques. Properly managed queues can minimize dropped packets and network congestion, as well as improve network performance. Thus, the queuing structure of CBWFQ (Class Based Weighted Fair Queuing) and strict Priority is combined so that the packets are classified into different classes and priorities are given to these classes, where the higher priority are given to delay sensitive packets and are scheduled effectively.
\end{abstract}

Keywords: Sliding mode control, Joint Congestion control and Scheduling, Network Utility Maximization, Dual Decomposition, Class Based Weighted Fair Queuing, Priority Queue, Lagrangian price.

\section{INTRODUCTION}

In recent years, there has been a progressive growth in the field of wireless networks. In Wireless networks, bandwidth is one of the major constraints. Some of the factors such as, mobility, random changes in connectivity, fluctuations in channel and interference due to neighboring nodes etc, leads to higher rate of packet loss in a wireless network than that of a wired network where, the packet loss occurs mainly due to congestion in the network.

The proper congestion control mechanism is to be incorporated in order to improve the performance of any network. So, the congestion control is required to control the rate of various traffic sources which inject the traffic into the network [18]. The systematic approach to analyze and design a system, predict system response to some input and approaches to assess system stability has been provided by some control theory. In wireless networks congestion control and scheduling are the key features that are to be considered. The joint congestion control and scheduling in wireless network can be represented by NUM problem which calculates the sum of link prices [1]-[3].

Sliding Mode (SM) control theory [18] is a powerful tool that can be used for congestion control. This SM controller can be applied to wireless networks by representing this joint congestion control and scheduling as NUM problem. The decomposition theory applied to the NUM problem facilitates us to apply the SM control technique to congestion control in ad hoc networks.
The rest of this paper is organized as follows. Section II, describes the system model, followed by the formulation and solution to the NUM problem, Section III extends the solution to include queuing structures. Section IV shows simulation results and section $\mathrm{V}$ concludes this paper.

\section{System Model}

\subsection{Queuing Structure}

The commonly used queuing structures are: 1) perdestination queues 2) per-link queues. For the networks that has per-destination queue, a separate queue is maintained for every node for each flow. The number of queues per node is equal to the number of nodes in the network. Overhead of such networks would be unbearable. Whereas, in per-link queuing networks the number of queues is based on the number of nodes in the next-hop that is every node needs to maintain a queue for every out going link attached with that node.

\subsection{Joint Congestion Control and Scheduling}

In a network the flow of information is accomplished through the interaction among different design layers in order to support transfer of information. In wireless networks, this interaction among the layers can be given by cross-layer design, where a number of parameters are jointly controlled. Furthermore, state information associated with a specific layer becomes available across layers [10].

Congestion control, routing and scheduling are implemented independently at different layers in the layered structure of 
the networks. However, in wireless networks, Congestion control and scheduling would be jointly designed to achieve high end-to-end throughput and efficient resource utilization [14].

\subsection{Network Utility Maximization}

The joint congestion control and scheduling in wireless networks is challenging due to unreliability, time varying channel and interference among wireless channels. Therefore the Network Utility Maximization (NUM) problem can be used to formulate the joint congestion control and scheduling [3].

Let us assume that a network has a set of resources as $\mathrm{L}$ and a set of users as I. Let $C_{l}$ denote the finite capacity of resource $l \in L$. Each user $i \in \mathrm{I}$ has a fixed route $r_{i}$ in which each user i's packet utilize the set of resources. $x_{i}(t)$ is the sending rate of source. In general, consider zero-one matrix $A$ which is defined, where $A_{i, l}=1$ if $l \in r_{i}$ and $A_{i, l}=0$ otherwise. When its rate is $x_{i}$ user i receive utility $U_{i}\left(x_{i}\right)$. The utility functions of the users are used to select the desired rate allocation among the users. The utility $U_{i}\left(x_{i}\right)$ is an increasing, strictly concave and continuously differentiable function.

$$
\max \Sigma U_{i}\left(x_{i}\right)
$$

Such that,

$$
\begin{aligned}
& A^{T} x \leq C \\
& x_{i, j}-F_{i, j}^{0}+F_{i, j}^{i n}
\end{aligned}
$$

Where, $C=C_{l} \in L$ is the capacity constraint which states that the sum of the rates of all users utilizing resource should not exceed its capacity $C_{l}$.

The joint congestion control and scheduling representation using NUM problem [3],[15] can be given as,

$$
\max \Sigma \mathrm{U}_{\mathrm{i}}\left(\mathrm{x}_{\mathrm{i}}\right)-\sum_{\mathrm{i}, \mathrm{j}} \in \mathrm{L} \lambda_{\mathrm{i}, \mathrm{j}}\left(\mathrm{x}_{\mathrm{i}, \mathrm{j}}-\mathrm{F}_{\mathrm{i}, \mathrm{j}}^{0}+\mathrm{F}_{\mathrm{i}, \mathrm{j}}^{\mathrm{in}}\right)
$$

\subsection{Dual Decomposition}

Dual decomposition decomposes the original large problem into distributive sub problems. This is mainly based on decomposing the Lagrangian dual problem.

This method of decomposition corresponds to a resource allocation with pricing. The original problem sets the price for the resources to each sub problem, depending on which it has to decide the amount of resources to be used.

From the NUM problem the congestion problem and scheduling problem can be decomposed separately as follows

$$
\max \Sigma \mathrm{U}_{\mathrm{i}}\left(\mathrm{x}_{\mathrm{i}}\right)-\sum_{\mathrm{i}, \mathrm{j}} \in \mathrm{L} \lambda_{\mathrm{i}, \mathrm{j}}\left(\mathrm{x}_{\mathrm{i}, \mathrm{j}}\right)+\max \sum_{\mathrm{i}, \mathrm{j}} \in \mathrm{L} \lambda_{\mathrm{i}, \mathrm{j}}\left(\mathrm{F}_{\mathrm{i}, \mathrm{j}}^{0}-\mathrm{F}_{\mathrm{i}, \mathrm{j}}^{\mathrm{in}}\right)
$$

\subsection{Distributed Hop-By-Hop Algorithm}

Necessity for full utilization of the potential capacity of the network arises due to the scarcity of the wireless spectrum. One approach to improve the capacity of a wireless network is to use multi-hop instead of traditional single-hop communication [14]. A distributed hop-by-hop algorithm is developed for congestion control. The congestion controller at the source reacts based on the sum of the congestion prices at each node. In other words, each node adds its current congestion cost to that it received from a downstream node, and passes this information toward the upstream node. The source will ultimately receive the sum of all price information from the corresponding nodes and use the information for controlling rates.

\subsection{Sliding Mode Based Control}

A sliding surface is constructed for switching surfaces so that the system restricted to the switching surface produces a desired behavior. For convenience only linear switching surfaces of the form $S_{x}(\mathrm{t})=0$ are considered in practice.

For sliding mode controller switched feedback gains which drive the state trajectory to the sliding surface and maintain it there. For the existence of a sliding mode on the switching surface, the state velocity vectors should be directed towards the surface, i.e., the system must be stable to the switching surface. Therefore a Lyapunov function can be used for maintaining the stability. The behavior of the system on the sliding surface is given as

$$
L(x, \lambda, v)=f(x)+\sum_{i} \lambda_{i} f_{i}(x)+\sum_{j} v_{j} h_{j}(x)
$$

Where, $\lambda_{i}$ is Lagrangian price [1], this lagrangian price can be updated according to

$$
\begin{gathered}
\lambda_{i, j}(t)=\left[y_{i, j}(X, t)\left(x_{i, j}-F_{i, j}^{0}+F_{i, j}^{i n}\right)\right] \\
\forall i, j \in L
\end{gathered}
$$

This lagrangian price value is proportional to the queuing length (or) delay in the node [2].

Each of the input flow $F_{i, j}^{i n}$ and output flow $F_{i, j}^{0}$ is obtained by estimating some parameters like arrival rate, transmission rate, and receive rate. The sliding mode controller is designed to adjust these parameters for stable behavior of the system, which in turn provides the feedback to control the delay value by maintaining the flow rate to a stable value by which congestion can be controlled [2].

Since the scheduling problem is given as

$$
\sum_{i, j} \in L
$$

It is seen that the scheduling also depends in some way on $\lambda_{i, j}(t)$ value. Hence by changing this value the packets would be scheduled properly. 


\subsection{Design of Sliding Mode Control Algorithm}

Each user $i$ adjusts its rate according to the following differential equation.

$$
\frac{d}{d t} x_{i}(t)=k_{i}\left[\omega_{i}-x_{i}(t) \sum_{l \epsilon r_{i}} p_{l}\left[\sum_{i \in I} x_{i}(t)\right]\right]
$$

Where, $\mathrm{k}_{\mathrm{i}}$ and $\omega_{\mathrm{i}}$ are positive constants, $k_{i}$ is the gain parameter, $\omega_{i}$ shows the user's willingness to pay per unit time. $\mathrm{P}(\mathrm{t})$ is an increasing function of the aggregate rate of the users going through it, and it can also be seen as the packet loss function.

The simplified dynamic model is

$$
r(t)=k(\omega-r(t) p(t))
$$

The dynamic buffer length at bottleneck is that

$$
\mathrm{q}(\mathrm{t})=\mathrm{r}(\mathrm{t})-\mathrm{C}
$$

Where, $\mathrm{q}(\mathrm{t})$ is the instantaneous queue length in buffer, $C$ is link capacity.

Let $\mathrm{x}_{1}(\mathrm{t})=\mathrm{q}(\mathrm{t})-\mathrm{q}_{\mathrm{d}}$ and Let $\mathrm{x}_{2}(\mathrm{t})=\mathrm{r}(\mathrm{t})-\mathrm{C}$

This can be also given as,

$$
\begin{aligned}
& \mathrm{x}_{1}(\mathrm{t})=\mathrm{x}_{2}(\mathrm{t}) \\
& \mathrm{x}_{2}(\mathrm{t})=\mathrm{k}\left[\omega-\left(\mathrm{x}_{2}(\mathrm{t})+\mathrm{C}\right)\right] \mathrm{p}(\mathrm{t})
\end{aligned}
$$

Where $q_{d}$ is the reference queue length

The queue length $q(t)$ and $p(t)$ is the marker probability. The queue length at congested routers is compared with the reference value $q_{d}$ and the feedback is provided. Then high link utilization and low delay is maintained in the system.

\section{PROPOSED SYSTEM}

The purpose of congestion control is to maintain and control the flow of packets that passes through the interface based on the individual priority bits assigned to each packets. This difficulty is overcome by effective classification and scheduling of packets and each are assigned to the newly created queue. There are four types of queuing protocols which helps in valuable congestion management by creating a different number of queues and different traffic management by varying the sequence in which the packets are transformed.

A network is congested when packets sending rate is faster than the transmission rate of the interface. By utilizing the congestion management schema, the packets are queued until the interface gets free and they are allowed to transfer from the corresponding interface based on the priority and the queuing mechanism assigned to that specific interface. The transmission among each queues are being done by the router which determines the order of packets to be transmitted and services that are provided by each queue.

The queuing structure which combines the feature of strict PQ (Priority Queue) and CBWFQ (Class Based Weighted Fair Queue) has been proposed.

CBWFQ defines classes with weights but does not provide strict priority. The traffic classes defined by CBWFQ are assigned with different characteristics. For example, the characteristic of a class can be the minimum bandwidth assigned during congestion [17].

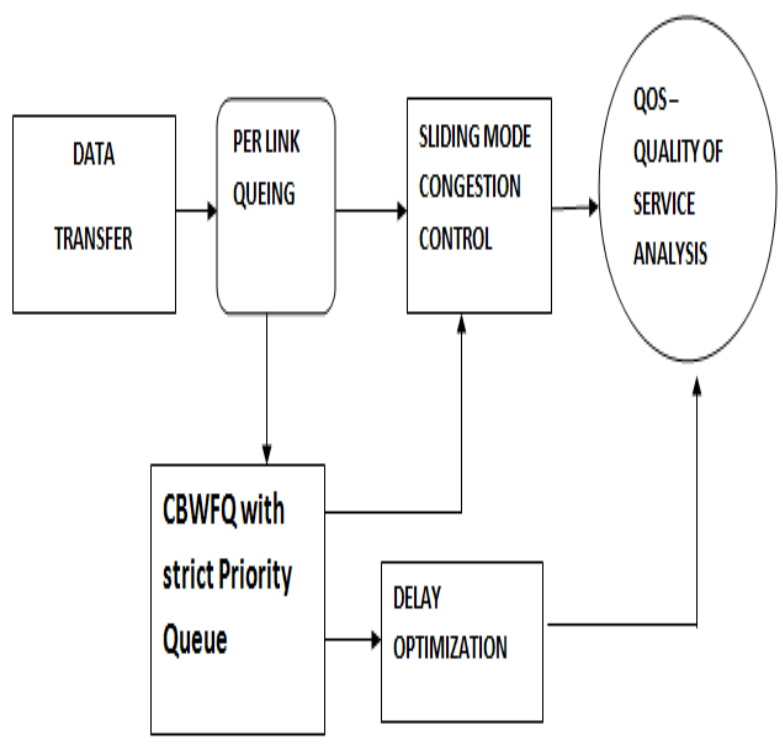

Fig. 3.1 Architecture Diagram

For CBWFQ, packets belonging to a specific class are given weights based on the bandwidth assigned to the class when configured. Therefore, the order in which packets are to be sent is determined by the bandwidth. Fair service is given to all packets based on weight.

Strict PQ allows delay-sensitive data such as voice to be dequeued and sent before packets in other queues are dequeued.

Thus by the proposed queuing structure the delay can be reduced further and the delay sensitive applications can be supported. 


\section{SIMULATION RESULTS}

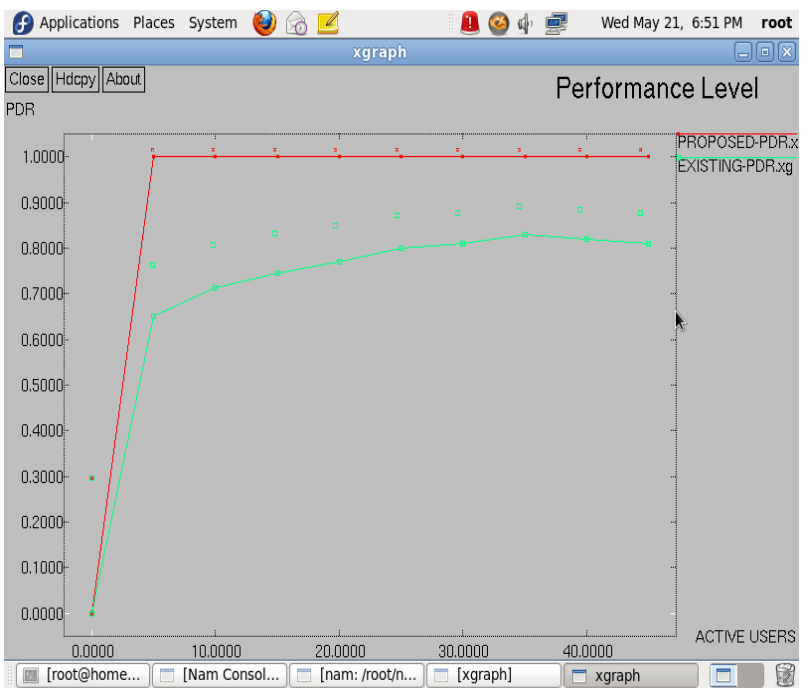

Fig. 4.1 Packet Delivery Ratio

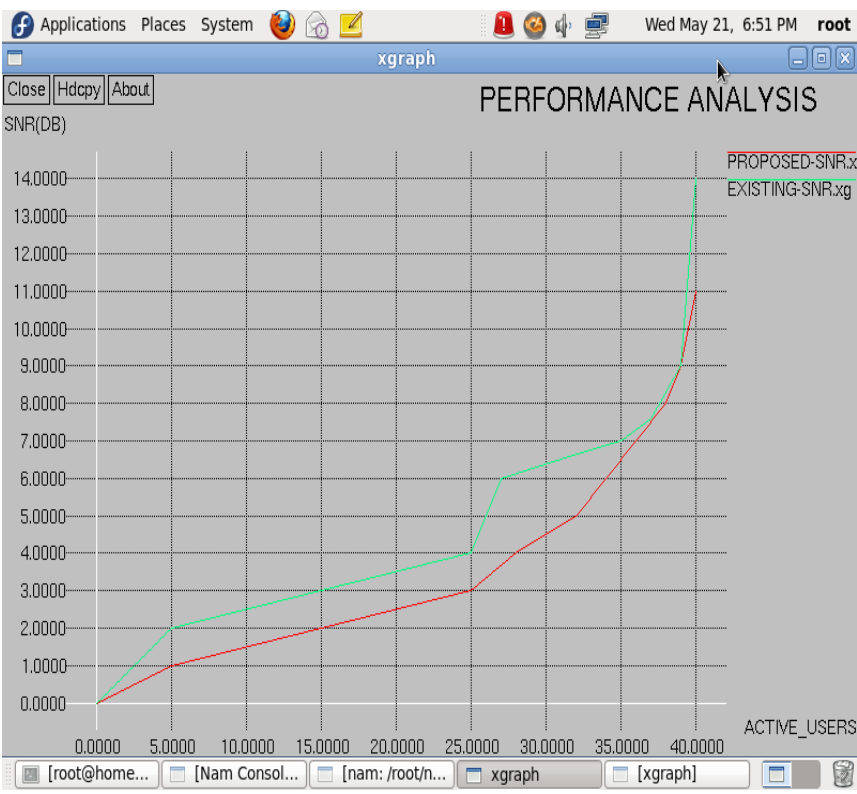

Fig. 4.2 Performance Based on SNR

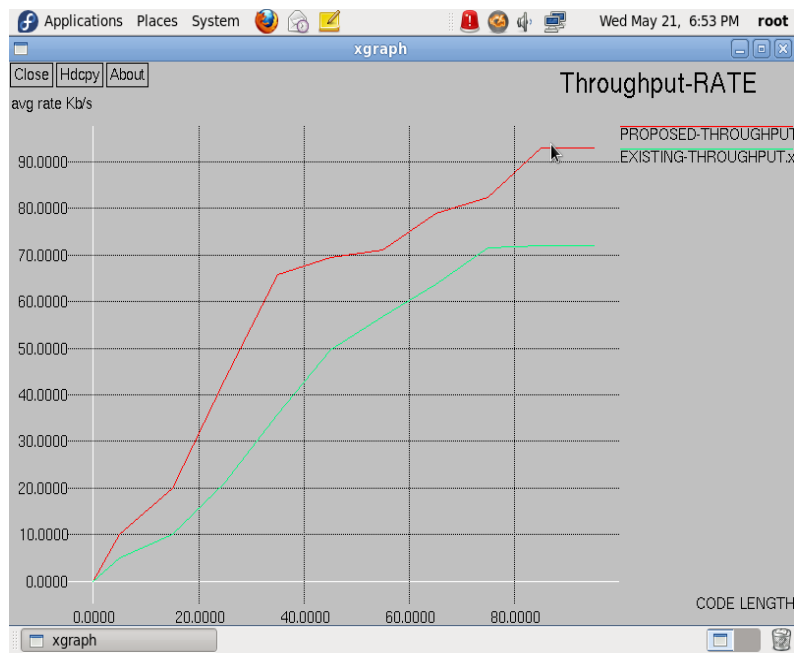

Fig. 4.3 Throughput Rate

\section{CONCLUSIONS}

In this paper, NUM problem is formulated to consider the joint congestion control and scheduling problem for multihop multipath per-link queuing wireless networks with QoS constraints. The NUM problem is decomposed and to solve the congestion control problem, a distributed sliding mode controller is designed to provide multipath rate adaptation to satisfy QoS constraints. Because of the multipath load balancing feature, it is robust against network anomalies such as link failure. Moreover queuing technique based on Class Based Weighted Fair Queuing is used and priority is added in order to schedule the packets of delay sensitive applications.

\section{REFERENCES}

[1]. Zongrui Ding and Dapeng Wu, "Sliding Mode Based Joint Congestion Control and Scheduling for Multiclass Traffic Over Per-Link Queuing Wireless Networks." IEEE/ACM Trans. on vehicular technology, vol. 62, no. 3, march 2013.

[2]. Yung Yi, Member, IEEE, and Sanjay Shakkottai, Member, IEEE "Hop-by-Hop Congestion Control Over a Wireless Multi-Hop Network", IEEE/ACM transactions on networking, vol. 15, no. 1, february 2007.

[3]. Zongrui Ding and Dapeng Wu, "Sliding Mode Based Joint Congestion Control and Scheduling in Multi hop Ad Hoc Networks with Multi-class Services"

[4]. Constantino M. Lagoa, Member, IEEE, Hao Che, and Bernardo A. Movsichoff, Student Member, IEEE "Adaptive Control Algorithms for Decentralized Optimal Traffic Engineering in the Internet", IEEE/ACM transactions on networking, vol. 12, no. 3, june 2004.

[5]. Daniel P. Palomar, Member, IEEE, and Mung Chiang, Member, IEEE. "A Tutorial on Decomposition Methods for Network Utility Maximization", IEEE journal on selected areas in communications, vol. 24, no. 8, august 2006.

[6]. Haozhi Xiong, Ruogu Li, Atilla Eryilmaz, and Eylem Ekici "Delay-Aware Cross-Layer Design for Network Utility Maximization in Multi-Hop Networks", IEEE journal on selected areas in communications, vol. 29, no. 5, may 2011.

[7]. M. Neely, "Universal scheduling for networks with arbitrary traffic, channels, and mobility," in Proc. IEEE 49th Conf. Decision Control, Atlanta, GA, 2010, pp. 1822-1829.

[8]. L. Chen, S. Low, M. Chiang, and J. Doyle, "Cross-layer congestion control, routing and scheduling design in ad hoc wireless networks", in Proc. IEEE 25th Int. Conf. Comput. Commun., Barcelona, Spain, 2006, pp. 1-13.

[9]. Nannan Zhang, Georigi M. Dimirovski, Yuanwei Jing, and Siying Zhang, "AQM Algorithm Based on Kelly's Scheme Using Sliding Mode Control" American Control Conference june 2009.

[10]. L. Georgiadis, M. Neely, M. Neely, and L. Tassiulas, "Resource Allocation and Cross Layer Control in Wireless Networks". Boston, MA: Now, 2006.

[11]. D. Palomar and M. Chiang, "Alternative distributed algorithms for network utility maximization: Framework and applications," IEEE Trans. Autom. Control, vol. 52, no. 12, pp. 2254-2269, Dec. 2007. 
[12]. M. Neely, "Dynamic power allocation and routing for satellite and wireless networks with time varying channels", Ph.D. dissertation, Mass. Inst. Technol., Cambridge, MA, 2003.

[13]. Z. Ding and D. Wu, "Capacity region and dynamic control of per next-hop queueing wireless networks under time varying channels", in Proc. INFOCOMM, 2011, pp. 110.

[14]. X. Lin and N. Shroff, "Joint rate control and scheduling in multihop wireless networks", in Proc. IEEE 43rd Conf. Decision Control, Paradise Island, Bahamas, 2004, pp. 1484-1489.

[15]. Y. Yi and M. Chiang, "Stochastic network utility maximization-attribute to Kelly's paper published in this journal a decade ago", Eur. Trans. Telecommun., vol. 19, no. 4, pp. 421-442, Jun.2008.

[16]. M. Neely, E. Modiano, and C. Rohrs, "Dynamic power allocation and routing for time-varying wireless networks," IEEE J. Sel. Areas Commun., vol. 23, no. 1, pp. 89-103, Jan. 2005.

[17]. Kampong J. Mabe, "LLQ in Integrated Services Networks", Rand Afrikaans University, Auckland Park, South Africa.

[18]. R. Barzamini1 Student Member, IEEE, M. Shafiee2, Member, IEEE, "A New Sliding Mode Controller for TCP Congestion Control", Journal. Basic. Appl. Sci. Res., 2(7)7175-7180, 2012

[19]. Fernando Paganini, Member, IEEE, Zhikui Wang, Student Member, IEEE, John C. Doyle, and Steven H. Low, Senior Member, IEEE, "Congestion Control for High Performance, Stability and Fairness in General Networks", IEEE/ACM Transactions on Networking, vol. 13, no. 1, February 2005. 